\title{
Effects of Nigella sativa on Plasma Oxidative Stress, and Some Apoptotic Protein Markers in Cerebrum and Hippocampus in Pentylenetetrazol-Induced Kindling Rats
}

\author{
Savas Ustunova' ${ }^{1}$, Ismail Meral ${ }^{1}$ (D) Abdurrahim Kocyigit ${ }^{2}$ (D), Aysu Kilic ${ }^{1}$ (D), \\ Oyku Zeybek ${ }^{1}$, Mehmet Uyuklu ${ }^{3}$, Sedat Meydan ${ }^{4}$ (D)
}

'Bezmialem Vakif University, School of Medicine, Department of Physiology, Istanbul, Turkey 2Bezmialem Vakif University, School of Medicine, Department of Biochemistry, Istanbul, Turkey ${ }^{3}$ Siirt University, School of Medicine, Department of Physiology, Siirt, Turkey

${ }^{4}$ Bezmialem Vakif University, School of Medicine, Department of Anatomy, Istanbul, Turkey

ORCID IDs of the authors: S.U. 0000-0003-1870-229X; I.M. 0000-0002-0973-4305; A.K. 0000-0003-2335-412X; A.K. 0000-0002-8593-1415; O.Z. 0000-0002-4554-3995; M.U. 0000-0002-7100-9817; S.M. 0000-0002-1393-3235

Please cite this article as: Ustunova S, Meral I, Kocyigit A,Kilic A, Zeybek O, Uyuklu M, Meydan S. Effects of Nigella sativa on Plasma Oxidative Stress, and Some Apoptotic Protein Markers in Cerebrum and Hippocampus in Pentylenetetrazol-Induced Kindling Rats. Eur J Biol 2020; 79(2): 124-131. DOI: 10.26650/EurJBiol.2020.0088

\begin{abstract}
Objective: This study was designed for investigating the effectiveness of the Nigella sativa (N. sativa) extract on apoptosis in cerebrum and hippocampus and also plasma oxidative stress, in pentylenetetrazol (PTZ)-induced kindling rats.

Materials and Methods: The kindling model was induced by subconvulsive doses ( $35 \mathrm{mg} / \mathrm{kg}$ i.p.) of intraperitoneal PTZ injections in N. sativa treated and non-treated PTZ groups (PTZ and PTZ + NS). The PTZ + NS group were also treated with an extract of $N$. sativa $(10 \mathrm{mg} / \mathrm{kg}) 2 \mathrm{~h}$ before each PTZ injection. The total oxidant and antioxidant status, oxidative stress index, paraoxonase, arylesterase, catalase (CAT) and total thiol levels were analyzed in plasma. Brain derived neurotrophic factor (BDNF), cyclin-B1 and B-cell lymphoma 2 (Bcl-2) expressions were investigated in the cerebrum and hippocampus.

Results: PTZ decreased the oxidative stress by increasing the activities of CAT, arylesterase, and paraoxonase. N. sativa decreased activities of arylesterase, paraoxonase, while increasing the CAT. It also brought the decreased BDNF and Bcl-2 expression levels to their normal levels in the cerebrum but not in the hippocampus.
\end{abstract}

Conclusion: $N$. sativa treatment improved the PTZ induced-impairments in BDNF and Bcl-2 expressions, resulting in a neuronal apoptosis in the cerebrum, without affecting blood oxidative stress.

Keywords: Apoptosis, epilepsy, cerebrum, hippocampus, N. sativa, oxidative stress

\section{INTRODUCTION}

Epilepsy is a neurological disease which affects millions of people with different ages worldwide (1). In recent years, many third-generation anti-epileptic drugs have been introduced. However, anti-epileptic drugs have some side effects including nausea, dizziness, and somnolence (2). Due to the fact that anti-epileptic drugs have some side effects and fail to control seizures in some patients, new therapies with lesser side effects have been investigated by researchers. Plant-origin therapies are especially popular nowadays due to their minimal side effects. Among them, Nigella sativa (N. sativa) is regarded as a kind of miracle herb since many scientists have revealed its vast spectrum of pharmacological potentials (3). In particular, studies on antiepileptic effects of $N$. sativa or one of its active constituents, thymoquinone, are popular. In many previous studies, the potent anti-epileptic role of $N$. sativa was investigated $(4,5)$. 
Kindling is one of the most studied and well-understood chronic animal models of epilepsy, generated either by a chemical or an electrical stimulus which is applied in subconvulsive doses repetitively and finally causes the generation of a chronic epilepsy model. Regular administration of pentylenetetrazol (PTZ) in subconvulsive doses triggers convulsions by causing decreased inhibitory or increased excitatory effects, which is called PTZ kindling (6).

Oxidative stress can trigger remarkable damage in the nervous system. Production of reactive oxygen species (ROS) in particular may cause multiple neuronal injuries that include lipid destruction of the cellular membrane, the cleavage of DNA, cell membrane lipid peroxidation and protein oxidation. In addition to the destruction of cellular integrity, ROS may also interrupt mitochondrial respiration (7). The endogenous antioxidant enzymes, such as catalase (CAT), arylesterase, paraoxonase and thiol are vital for compensating oxidative stress (8-10). Their amounts in serum or tissue are important for elucidating the oxidant-antioxidant balance. It has been shown that $N$. sativa, which contains many antioxidant compounds such as thymoquinone, has a neuroprotective effect on the brain by reducing oxidative stress (11).

Brain derived neurotrophic factor (BDNF) is one of the most important growth factors in the central nervous system (CNS). It has a few different biologically active isoforms, which also interact with different receptor types (12). It is vital for the development of the CNS and for brain plasticity (13). Due to the fact that BDNF plays a crucial role in CNS, it has close relationships with any disease that affects the brain, such as epilepsy. The B-cell lymphoma 2 ( $\mathrm{Bcl}-2)$ protein family has vital roles in cells, especially in the process of apoptosis. Bcl-2 itself is an anti-apoptotic protein from the $\mathrm{Bcl}-2$ family and its amount is directly proportional to the vitality of the cell. Increment levels in the $\mathrm{Bcl}-2$ protein lead to the survival of the cell via inhibiting apoptosis. Additionally, cyclins and cyclin-dependent kinases, CDKs, are the basic regulators for the cell cycle and their amount regulates cell-cycle events, such as neurogenesis, progression of cell to apoptosis and maintaining the health of CNS and are also shown to take part in many pathophysiological process in CNS including epilepsy (14).

Therefore, this study was planned for investigating the effects of $N$. sativa extract on plasma oxidative stress, and some apoptotic and cell-cycle protein markers in cerebrum and hippocampus in PTZ-induced kindling rats.

\section{MATERIALS AND METHODS}

\section{Chemicals}

A physiological saline solution was used to dissolve the PTZ (Sigma-Aldrich Co., St. Louis, MO, USA). PTZ was injected intraperitoneally (i.p). N. sativa seeds were obtained from a local herbal market. The seeds were ground by a classical grinder (MC 23200, Siemens-Elektrogeräte $\mathrm{GmbH}$, Hamburg, Germany), and its methanolic extract was papered as described previously (15). Four ml sterile physiological saline solution was used to dissolve $10 \mathrm{mg}$ of lyophilized extract to have a stock solution which then was administered to animals by gastric gavage in adequate volumes.

\section{Animals and Experimental Design}

Twenty one male adult Wistar albino rats (230-300 g) were used in our study. The rats were obtained from Bezmialem Vakif University's Experimental Animal Centre and housed under standard temperature $\left(25 \pm 1^{\circ} \mathrm{C}\right)$, humidity $(50-60 \%)$, dark-light conditions (12 h light/12 h dark cycle) and fed ad libitum. Ethical approval was obtained from the Bezmialem Vakıf University Laboratory Animals Ethics Committee. The rats were divided into 3 groups: a control group $(\mathrm{C}, \mathrm{n}=7)$; PTZ treated $(\mathrm{PTZ}, \mathrm{n}=7)$ and $\mathrm{PTZ}+N$. sativa treated (PTZ + NS, $n=7)$. PTZ-kindling model was achieved for the PTZ and PTZ + NS groups by multiple PTZ injections in a subconvulsive dose of $35 \mathrm{mg} / \mathrm{kg}$ on days $1,3,5,8,10,12,15,17$, 19,22 and 24 of the study. Ten $\mathrm{mg} / \mathrm{kg}$ methanolic extract of $N$. sativa was administered to the rats in the PTZ + NS group by oral gavage, just 2 hours before PTZ injection. The control group was only treated with $4 \mathrm{ml} / \mathrm{kg}$ saline. Seizures were observed after 30 minutes following PTZ administration. After establishment of the kindling model, convulsive doses of PTZ (75 mg/kg) were injected to the PTZ and PTZ + NS groups to induce tonic and clonic seizures on the $26^{\text {th }}$ day of the study. The control group was not injected with PTZ. For the PTZ + NS group, convulsive doses of PTZ were injected 2 hours after $N$. sativa extract $(10 \mathrm{mg} / \mathrm{kg}$ ) administration. For describing the characteristics of the behavioral seizure, a modified scale was used (data not shown) (16). Upon the completion of the experiment, the rats were anesthetized (15 mg/kg xylazine and $50 \mathrm{mg} / \mathrm{kg}$ ketamine), decapitated, and the hippocampi and cerebrum were dissected for Western blot and biochemical analysis. The samples were stored at $-80^{\circ} \mathrm{C}$.

\section{Blood Sampling}

The blood was drawn by cardiac puncture into heparinized tubes, and stored at $+4^{\circ} \mathrm{C}$. The blood samples were centrifuged at $3.500 \mathrm{x}$ $\mathrm{g}$ for 5 minutes. The plasma samples were stored at $-80^{\circ} \mathrm{C}$ until the analysis of paraoxonase, arylesterase and CAT activities, and total thiol, total oxidant and antioxidant status (TOS and TAS) levels.

\section{Analysis of Oxidant Markers}

\section{Total Oxidant and Antioxidant Status}

The TOS and TAS levels were measured by commercial kits using spectrophotometric methods according to manufacturer instruction (Rel Assay Diagnostics, Gaziantep, Turkey). A 1420 Victor 3 Multilabel Plate Reader was used for the spectrophotometric analysis (PerkinElmer, Waltham, MA, USA). The test results of TOS were demonstrated in terms of $\mu \mathrm{m} \mathrm{H}_{2} \mathrm{O}_{2}$ Equiv/ $L$, and the test results of TAS were demonstrated in terms of $\mu \mathrm{m}$ Trolox Equiv/L.

\section{Calculation of Oxidative Stress Index (OSI)}

Following the measurement of TOS and TAS values, the results were calculated according to the formula below and demonstrated as an arbitrary unit.

oxidative Stress Index (OSI) $=\frac{\operatorname{TOS}\left(\mu \mathrm{mol} . \mathrm{H}_{2} \mathrm{O}_{2} \cdot \text { Equivalent } / L\right)}{\operatorname{TAS}(\mu \mathrm{mol} \text {.Trolox. Equivalent } / L)} \times 100$

\section{Paraoxonase and Arylesterase Activities}

The activities of paraoxonase and arylesterase were assayed 
by a commercial kit (Rel Assay Diagnostics, Gaziantep, Turkey). Two different substrates were used for the paraoxonase-1 activity. Owing to the formation of p-nitrophenol, the paraoxon hydrolysis rate was calculated by recording the increment in absorbance at $412 \mathrm{~nm}, 25^{\circ} \mathrm{C}$. Paraoxonase- 1 activity was defined as $1 \mathrm{mmol}$ p-nitrophenol generated per minute under standard conditions and demonstrated as U/L (17). Arylesterase activity was demonstrated in terms of $\mathrm{kU} / \mathrm{L}$ and it was identified as 1 mmol of phenol generated per minute under standard reaction conditions. No enzyme was added to blanks and they were used to correct the spontaneous hydrolysis of both substrates (18).

\section{Catalase Activity}

A gasometric procedure, which measures the amount of oxygen produced by the decomposition of $\mathrm{H}_{2} \mathrm{O}_{2}$ was performed for assessing CAT activity. The activity of CAT was established by the decrease in $\mathrm{H}_{2} \mathrm{O}_{2}$ absorbance at $240 \mathrm{~nm}$. A multiplate reader was used for assessment (Varioskan Flash Multimode Reader; Thermo Fisher Scientific, Waltham, MA).

\section{Total Thiol Measurement}

Natural thiol and total thiol, as well as disulfide levels, were measured with a new automated method (19). The new method is based on the reduction of disulfide bonds to thiol groups and sulfhydryl groups of proteins, converting them to the reversible disulfide form under oxidative conditions. The final diagnosis was approved by histopathological testing. The findings of the appendectomy sample histopathology were classified as non-perforated or perforated appendicitis.

\section{Western Blotting Assay}

The hippocampus and cerebrum regions of the brains were surgically removed and homogenized in a lysis buffer containing pro- tease inhibitor cocktails (MP FastPrep-24, MP Biomedicals, Irvine, CA USA). Then the homogenates were centrifuged at $14.000 \mathrm{x} \mathrm{rpm}$ (Beckman Coulter, Krefeld, Germany) at $4^{\circ} \mathrm{C}$ for 10 minutes and the cytosolic fraction was separated as the final supernatant. The Bradford assay was used to assess protein concentrations in the supernatants. A $5 \times$ loading buffer was added to the above-mentioned supernatant and boiled at $100^{\circ} \mathrm{C}$ for 15 minutes (19). Protein content was then separated on $8-12 \%$ sodium SDS-PAGE, transferred to a membrane made of PVDF, and then incubated with both primary and secondary antibodies conjugated with horseradish peroxidase (Cell Signaling Technology, Denvers, MA, USA). Separated protein bands were made visible by Pierce ECL Western blotting substrate (Thermo Fisher Scientific, Waltham, MA, USA) on secondary antibodies for BDNF, cyclin-B1, Bcl-2 and $\beta$-tubulin (Santa Cruz, Biotechnology, Dallas, TE, USA).

\section{Statistical Analysis}

All data were analyzed statistically using GraphPad Prism 6.0 (GraphPad Prism Software, San Diego, CA, USA), and demonstrated as mean \pm standard error of mean (mean \pm SEM). OneWay ANOVA and Student's $t$-test were used to analyze results in order to compare groups. The $p<0.05$ value was considered to be statistically significant.

\section{RESULTS}

\section{Oxidant Markers}

The plasma TAS, TOS and OSI levels of the study groups are shown in Figure 1. The plasma TOS levels of PTZ and PTZ + NS groups were significantly lower $(p<0.01)$ than controls (Figure $1 \mathrm{~B})$. Although the plasma OSI level was significantly $(p<0.05)$ lower in PTZ group, it was not statistically different $(p>0.05)$ in PTZ + NS group compared to controls (Figure 1C).
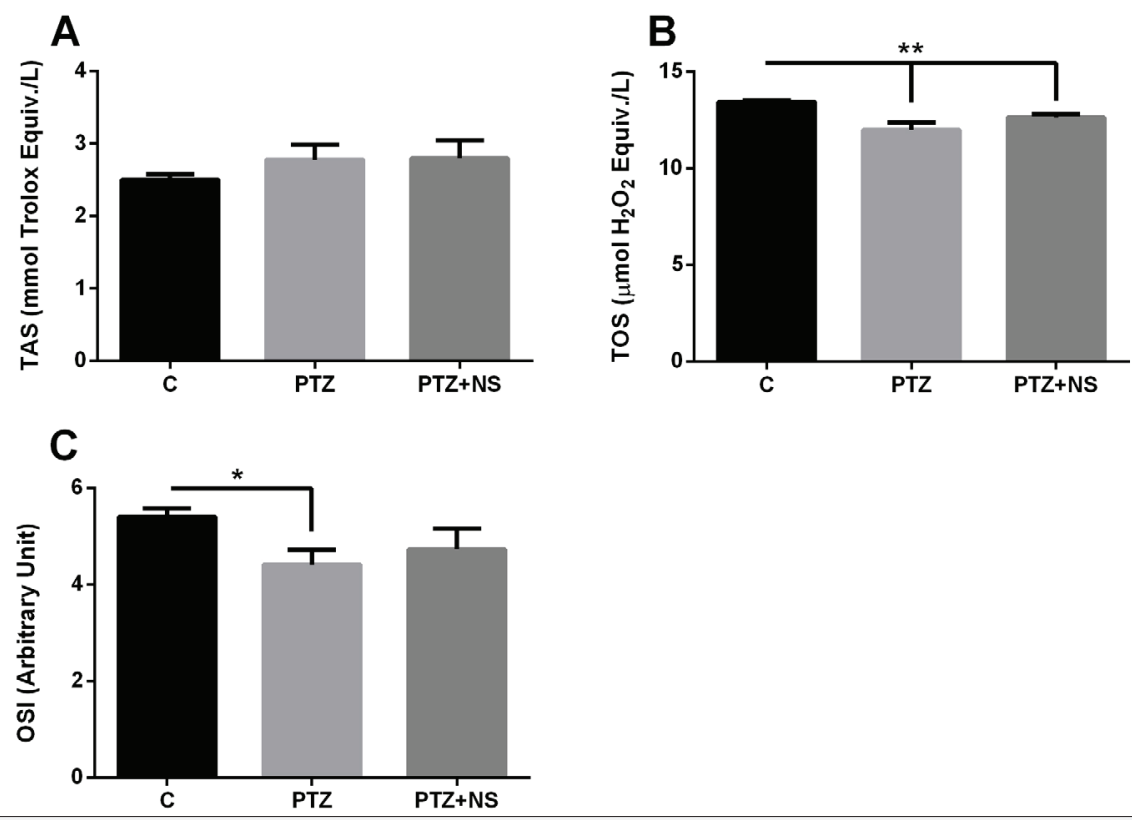

Figure 1. The plasma TAS (A), TOS (B) and OSI (C) levels of the study groups ( $n=7)$. Data is presented as mean \pm standard error of mean. TOS; Total Oxidant Status, TAS; Total Antioxidant Status, OSI; Oxidative Stress Index. ${ }^{*} ; p<0.05,{ }^{* *} ; p<0.01$. 

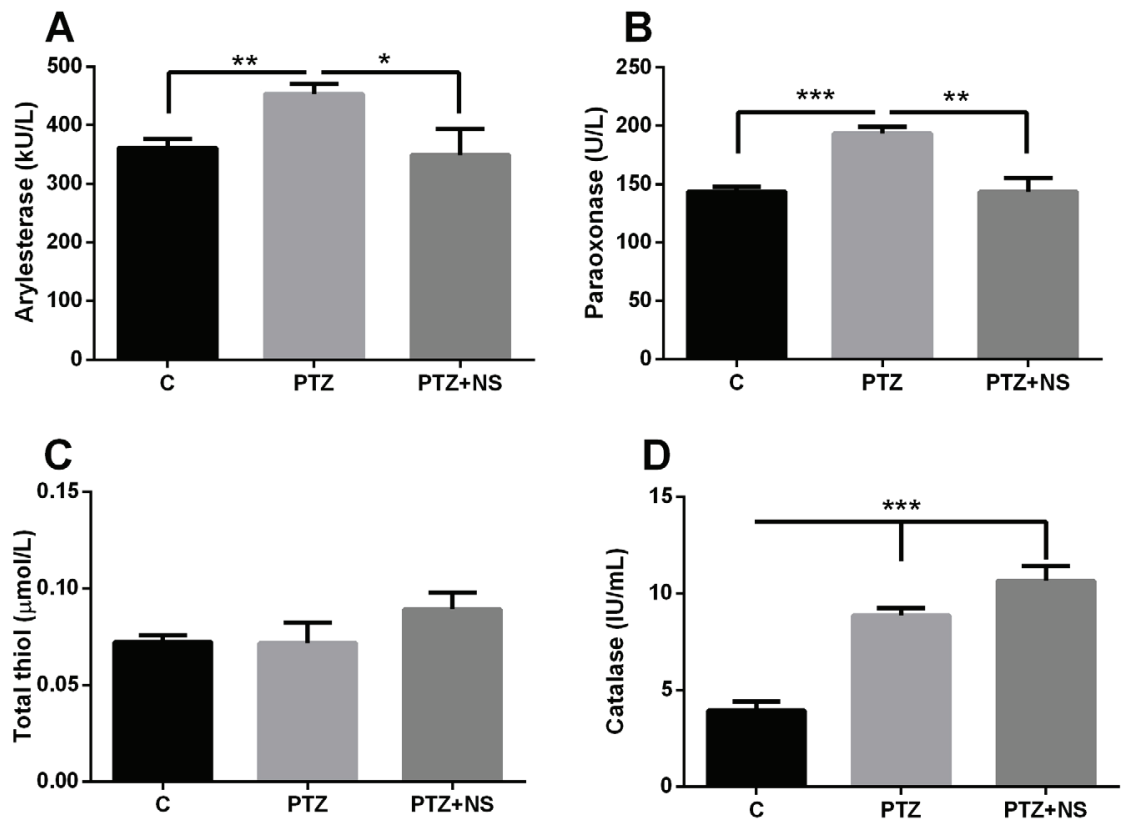

Figure 2. The plasma arylesterase (A), paraoxonase (B), total thiol $(C)$ and CAT (D) levels of groups $(n=7)$. Data is presented as mean \pm standard error of mean. CAT; Catalase. ${ }^{*} ; p<0.05,{ }^{* *} ; p<0.01,{ }^{* *} ; p<0.001$.

\section{Antioxidant Markers}

The plasma arylesterase, paraoxonase and CAT activities, and total thiol levels of groups are shown in Figure 2. The plasma arylesterase activity significantly $(p<0.01)$ increased in the PTZ group compared to the $C$ group. But it decreased $(p<0.05)$ in PTZ + NS group compared to PTZ group, there was no significant difference $(p>0.05)$ between the $C$ and PTZ + NS groups (Figure 2A). Similar results were also obtained regarding plasma paraoxonase activity. It significantly $(p<0.001)$ increased in
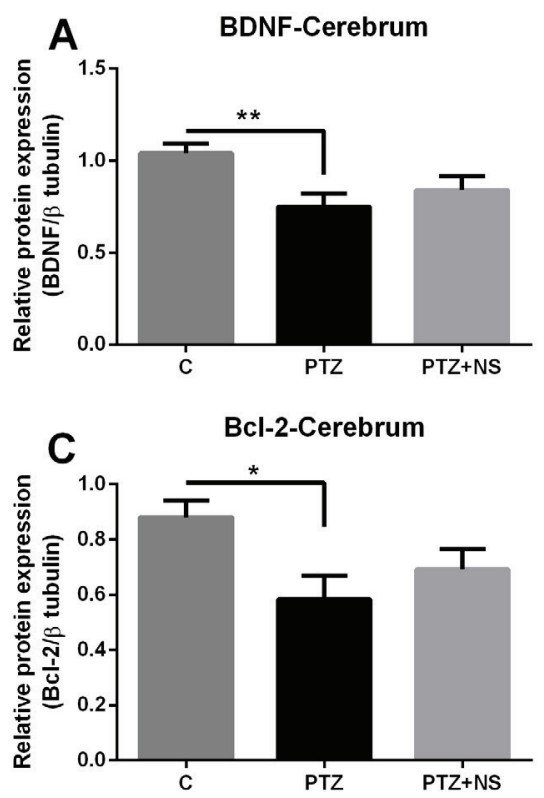

PTZ group compared to the $C$ group. But it decreased $(p<0.01)$ in the PTZ + NS group compared to PTZ group, there was no significant difference $(p>0.05)$ between the $C$ and PTZ + NS groups (Figure 2B). Although the plasma total thiol level tended to increase in the PTZ and PTZ + NS groups compared to the controls, it was not different ( $p>0.05$ ) among the groups (Figure $2 C)$. However, the plasma CAT activity significantly $(p<0.001)$ increased in both PTZ and PTZ + NS groups compared to the controls (Figure 2D).
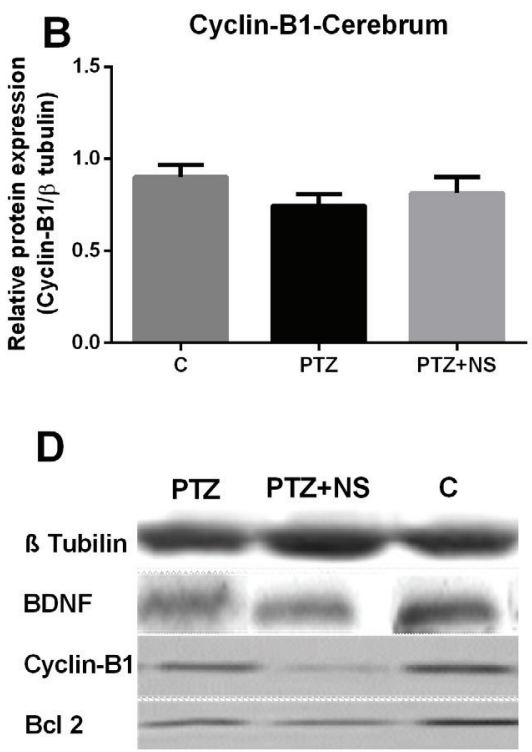

Figure 3. The Western blotting assay results of BDNF (A), Cyclin-B1 (B), Bcl-2 (C) and the image of related bands (D) in cerebrums of groups ( $n=7)$. Data is presented as mean \pm standard error of mean. BDNF; Brain derived neurotrophic factor, Bcl-2; $\mathrm{B}$-cell lymphoma $2 .{ }^{*} ; p<0.05,{ }^{* *} ; p<0.01$. 

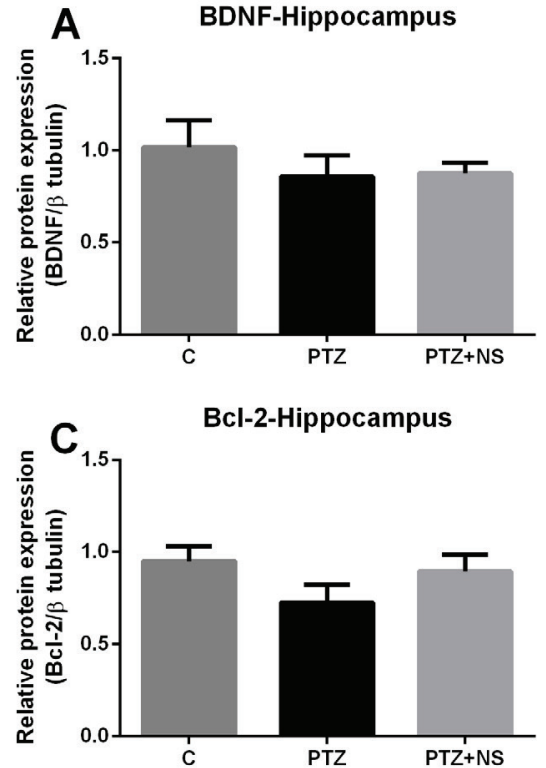
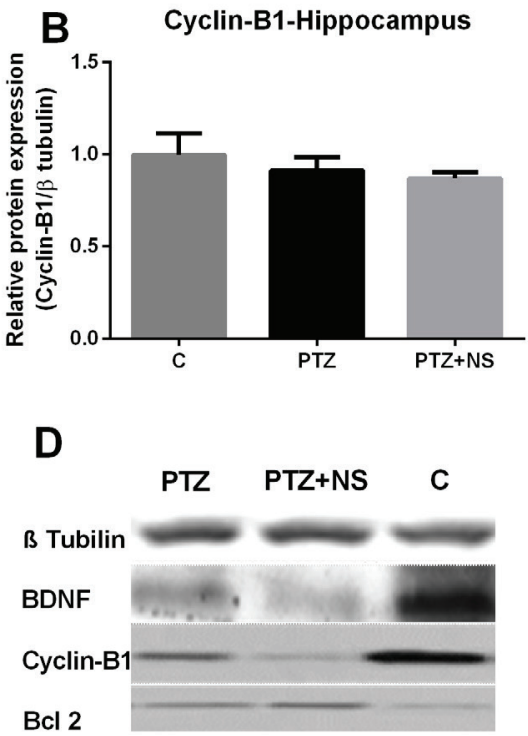

Figure 4. The Western blotting assay results of BDNF (A), Cyclin-B1 (B), Bcl-2 (C) and the image of related bands (D) in hippocampus of groups $(n=7)$. Data is presented as mean \pm standard error of mean. BDNF; Brain derived neurotrophic factor, Bcl-2; B-cell lymphoma 2.

\section{Western Blotting Assay}

Western blotting assay results of cerebrum are shown in Figure 3. The protein expression levels of BDNF (Figure $3 \mathrm{~A}$ ) and $\mathrm{BCl}-2$ (Figure $3 C$ ) significantly ( $p<0.01$ and $p<0.05$, respectively) decreased in the PTZ group compared to the controls. However, there was no significant ( $p>0.05$ ) difference between $C$ and PTZ + NS groups in terms of BDNF or Bcl-2 protein expression levels. In addition, the level of cyclin-B1 protein expression was not different ( $p>0.05$ ) among the groups (Figure 3B).

Western blotting assay results of hippocampus are shown in Figure 4. There was no statistical ( $p>0.05$ ) difference among groups regarding $\mathrm{BDNF}$, cyclin-B1 or $\mathrm{Bcl}-2$ protein expression levels.

\section{DISCUSSION}

This study is a part of our previously published study (20) and was designed for investigating the effects of $N$. sativa extract on some plasma oxidant and antioxidant markers in PTZ-induced kindling rats. It was found that the PTZ treatment decreased the plasma oxidative stress by increasing the activities of arylesterase, paraoxonase and CAT. It is well known that oxidative stress has a crucial role in the pathogenesis of epilepsy (21). Some studies indicated that oxidative stress increased in PTZ-induced epilepsy $(22,23)$. However, our findings contrast with the findings of previous studies in the literature. This might be due to high plasma antioxidant arylesterase, paraoxonase and CAT activities that reduced the oxidative stress in the PTZ group. Exogenous antioxidant $N$. sativa treatment, on the other hand, decreased the activities of endogenous antioxidant enzymes arylesterase and paraoxonase. To maintain homeostasis, when animal or human cells are subjected to mild biological stress, they activate cellular and physiological processes (24).
Increased production of endogenous antioxidant enzymes in response to mild biological stress protects the organism from intense oxidative stress produced by heavy metals, toxic substances or pro-oxidant compounds (25). Low doses of various stress producing agents may disturb the physiological parameters of an organism but may not induce severe damage. It may improve the ability of an organism to resist severe stresses by inducing adaptive responses, and has actually a beneficial effect, which is called hormesis (26). Hormetic response is formed when cells or organisms are exposed to mild biological stress, such as caloric restriction, exercise, environmental toxins and inorganic compounds (27). The dose of PTZ used in this experiment for 11 treatments was subconvulsive $(35 \mathrm{mg} / \mathrm{kg})$, and only the final ( $12^{\text {th }}$ treatment) was convulsive $(75 \mathrm{mg} / \mathrm{kg})$. Therefore, it might be said that the subconvulsive dose of PTZ produced a hormetic response that increased the antioxidants arylesterase, paraoxonase and CAT activities leading to a reduction in the oxidative stress in PTZ group compared to the controls.

Theoretically, intake of exogenous antioxidants should support the endogenous defence system by decreasing oxidative damage. However, in our study, exogenous antioxidant $N$. sati$v a$ treatment decreased the elevated-activities of endogenous antioxidants arylesterase and paraoxonase, with no net change in OSI level. The endogenous antioxidants are produced in the body, and many of them are encoded by the nuclear factor erythroid 2-related factor 2 (Nrf2) - keap1 (kelch-like ECH-associated protein 1) pathway $(28,29)$. It has been commonly suggested that thymoquinone, an active biological form of $\mathrm{N}$. sativa, serves antioxidant activity and reduces lipid peroxidation by increasing the production of antioxidant enzymes and the expression of Nrf2 in many tissues (30,31). Hamdan, Al-Gayyar (32) suggested that the exposure of rats' brain tissues to TQ 
ameliorated the sodium nitrite-induced oxidative stress, and increased the lowered Nrf2 expression and antioxidant enzymes. However, in our experiment, PTZ treatment did not induce oxidative stress or decrease in the antioxidant enzyme activities. Conversely, it lowered the oxidative stress by increasing the antioxidant enzyme activities. Subsequently, the $N$. sativa treatment decreased the elevated-activities of endogenous antioxidants arylesterase and paraoxonase in PTZ-treated rats. From the results of our present, and also previous studies it might be said that $N$. sativa had dual effects on antioxidant enzyme activities (20). It increases the antioxidant enzyme activity when their activities are low but it decreases the antioxidant enzyme activity when their activities are high. Similar to our finding, it has been reported that exogenous antioxidants may downregulate the endogenous protection, thus causing no net change in antioxidant defences (33).

In this study, the exogenous antioxidant $N$. sativa treatment increased the CAT level further. CAT is a ubiquitous antioxidant enzyme that is present in nearly all living organisms. It is active in the detoxification of $\mathrm{H}_{2} \mathrm{O}_{2}$ in human erythrocytes (34). Increased CAT activity by $N$. sativa treatment may be an attempt by the body to compensate for the low antioxidant (arylesterase and paraoxonase) effect, or may be a direct effect of $N$. sativa treatment. There are studies indicating that $N$. sativa treatment increased CAT activity $(35,36)$.

The second aim of this study was to examine the effects of $N$. sativa extract on the expression levels of some apoptotic proteins in both cerebrum and hippocampus in PTZ-induced kindling rats. In the cerebrum, the protein expression levels of $\mathrm{Bcl}-2$ and BDNF decreased but cyclin-B1 level did not change in the PTZ group compared to the controls. $N$. sativa extract treatment brought the levels of $\mathrm{Bcl}-2$ and BDNF to normal. BDNF is a small dimeric protein that is responsible for the growth of neurons. Its most common site is the hippocampus and the cerebral cortex. It has a protective effect on neurons and is associated with neuroplasticity (37). Decreased BDNF levels correlate with many neurodegenerative diseases that exhibit neuronal loss (38). A number of studies have shown that both BDNF and its specific receptor tropomyosin receptor kinase B (TrkB) expression increases in epilepsy models (39-43). Current studies suggest that serum BDNF is constantly higher in patients with epilepsy and appears to be associated with the pathology of the disease. Hong et al. indicated that the concentration of the serum BDNF is proportional with the severity of the disease (44). It has been reported that BDNF levels significantly decreased during the interictal period of epilepsy in patients with cerebral autoregulation and autonomic function impairment. Severe and long-term epilepsy may disrupt BDNF signalling, thus causing impaired cerebral autoregulation and autonomic dysfunction in patients with epilepsy. It has been demonstrated that acute administration of BDNF, both in vivo and in vitro, induces the neuronal excitability and facilitates epileptogenesis (45).

$\mathrm{Bcl}-2$ is a cell survival protein whose main role is to inhibit apoptosis by preventing the cytochrome $\mathrm{c}$ release and the activation of caspases (46). The levels, and also interactions of $\mathrm{Bcl}-2$ with other pro-apoptotic proteins are critical for the vitality of a neuron. Under certain cellular stress, Bcl-2/pro-apoptotic protein ratio decreases, leading to apoptosis (47). PTZ is a neurotoxic agent that causes neuronal stress and provokes kindling. Similarly, Shi et al. indicated that PTZ treatment decreased the expression of the $\mathrm{BCl}-2$ protein (22). The decreased expressions of $\mathrm{Bcl}-2$ and BDNF in the cerebrum of PTZ-treated rats indicated an increased apoptosis. The $N$. sativa treatment reversed the decreased-expression of $\mathrm{Bcl}-2$ and BDNF to normal levels, indicating the prevention of tissue from apoptosis. Hosseini et al. demonstrated that thymoquinone, an active compound of $N$. sativa, may decrease $\mathrm{Bax} / \mathrm{Bcl}-2$ ratio, thus reducing the severity of apoptosis under stress conditions, such as ethanol toxicity (48). Ullah et al. demonstrated that PTZ treatment decreased the expression of $\mathrm{Bcl}-2$ and pre-treatment with thymoquinone reversed that decrement by upregulating $\mathrm{Bcl}-2$ and also downregulating pro-apoptotic Bax (49).

Cyclin-B1 is among the most important core cell-cycle regulators. There is a link between the cell-cycle and neuronal death. Neuronal cell death that was accompanied by cyclin-B1 expression was reported in the hippocampal field of PTZ-kindled rats (50). Although BDNF and Bcl-2 expressions in the cerebrum were affected as indicated before, cyclin-B1 expression was not affected by PTZ-treatment in our study.

We obtained different results in hippocampus than those in the cerebrum, regarding expressions of $\mathrm{Bcl}-2$ and BDNF proteins in PTZ-treated rats. Although their expression levels were not different in groups of hippocampus tissues, they decreased in cerebrum tissues of PTZ-treated rats. This indicated that apoptosis occurred in the cerebrum but did not occur in the hippocampus of PTZ-treated rats. These results are similar to the results of our previous study, which demonstrated that PTZ treatment caused a neuronal loss due to apoptosis in the cerebral cortex, but not in the hippocampus (20). From the results of both studies, it might be said that apoptotic neuron numbers of the cerebrum increases in PTZ-kindling epileptic rats due to the decreased expression of $\mathrm{BCl}-2$ and BDNF proteins. The $N$. sativa brought the decreased-expression levels of $\mathrm{Bcl}-2$ and BDNF back to normal.

\section{CONCLUSION}

It was concluded that the $N$. sativa treatment improved the PTZ-induced impairments in BDNF and $\mathrm{BCl}-2$ expressions that reflect a neuronal apoptosis in the cerebrum, without affecting blood oxidative stress.

Peer-review: Externally peer-reviewed.

Author Contributions: Conception/Design of study: S.U., I.M.; Data Acquisition: S.U., I.M., A.K., A.K., O.Z., M.U.; Data Analysis/Interpretation: S.U., I.M., A.K., A.K., O.Z., M.U.; Drafting Manuscript: S.U., I.M., A.K., O.Z.; Critical Revision of Manuscript: S.U., I.M., A.K., M.U., S.M.; Final Approval and Accountability: S.U., I.M.; Technical or Material Support: S.U., I.M.; Supervision: S.U., I.M. 
Conflict of Interest: The authors declare that they have no conflicts of interest to disclose.

Financial Disclosure: This work was supported by the Research Fund of Bezmialem Vakif University [grant number 6.2013/8].

\section{REFERENCES}

1. Stafstrom CE, Carmant L. Seizures and epilepsy: an overview for neuroscientists. Cold Spring Harbor Perspect Med 2015; 5(6): a022426.

2. Anderson J, Moor C-C. Anti-epileptic drugs: a guide for the non-neurologist. Clin Med (Lond) 2010; 10(1): 54-8.

3. Ahmad A, Husain A, Mujeeb M, Khan SA, Najmi AK, Siddique NA, et al. A review on therapeutic potential of Nigella sativa: A miracle herb. Asian Asian Pac J Trop Biomed 2013; 3(5): 337-52.

4. Hosseinzadeh $\mathrm{H}$, Parvardeh $\mathrm{S}$, Nassiri-Asl M, Mansouri M-T. Intracerebroventricular administration of thymoquinone, the major constituent of Nigella sativa seeds, suppresses epileptic seizures in rats. Med Sci Monit 2005; 11(4): BR106-BR10.

5. Ilhan A, Gurel A, Armutcu F, Kamisli S, Iraz M. Antiepileptogenic and antioxidant effects of Nigella sativa oil against pentylenetetrazol-induced kindling in mice. Neuropharmacology 2005; 49(4): 456-64.

6. Dhir A. Pentylenetetrazol (PTZ) kindling model of epilepsy. Curr Protoc Neurosci 2012; 58(1): 9-37.

7. Radad K, Rausch W-D, Gille G. Rotenone induces cell death in primary dopaminergic culture by increasing ROS production and inhibiting mitochondrial respiration. Neurochem Int 2006; 49(4): 379-86.

8. Chaudière J, Ferrari-lliou R. Intracellular antioxidants: from chemical to biochemical mechanisms. Food Chem Toxicol 1999; 37(9-10): 949-62.

9. Haley RW, Billecke S, La Du BN. Association of low PON1 type Q (type A) arylesterase activity with neurologic symptom complexes in Gulf War veterans. Toxicol Appl Pharmacol 1999; 157(3): 227-33.

10. Hernández AF, Gil F, Lacasaña M, Rodríguez-Barranco M, Gómez-Martin A, Lozano $D$, et al. Modulation of the endogenous antioxidants paraoxonase- 1 and urate by pesticide exposure and genetic variants of xenobiotic-metabolizing enzymes. Food Chem Toxicol 2013; 61: 164-70.

11. Hobbenaghi R, Javanbakht J, Sadeghzadeh S, Kheradmand D, Abdi F, Jaberi M, et al. Neuroprotective effects of Nigella sativa extract on cell death in hippocampal neurons following experimental global cerebral ischemia-reperfusion injury in rats. J Neurol Sci 2014; 337(1-2): 74-9.

12. Lucini $C$, D'Angelo L, Cacialli P, Palladino A, de Girolamo P. BDNF, brain, and regeneration: Insights from zebrafish. Int J Mol Sci 2018; 19(10): 3155.

13. Autry $A E$, Monteggia $L M$. Brain-derived neurotrophic factor and neuropsychiatric disorders. Pharmacol Rev 2012;64(2):238-58.

14. Wolgemuth DJ, Manterola M, Vasileva A. Role of cyclins in controlling progression of mammalian spermatogenesis. Int J Dev Biol 2013; 57: 159.

15. Ali BH, Blunden G. Pharmacological and toxicological properties of Nigella sativa. Phytotherapy Research: An international journal devoted to pharmacological and toxicological evaluation of natural product derivatives. Phytother Res 2003; 17(4): 299-305.

16. Eraković V, Župan G, Varljen J, Laginja J, Simonić A. Altered activities of rat brain metabolic enzymes caused by pentylenetetrazol kindling and pentylenetetrazol—induced seizures. Epilepsy Res 2001; 43(2): 165-73.

17. Eckerson HW, Wyte CM, La Du B. The human serum paraoxonase/ arylesterase polymorphism. Am J Hum Genet 1983; 35(6): 1126.
18. Haagen L, Brock A. A new automated method for phenotyping arylesterase (EC 3.1.1.2) based upon inhibition of enzymatic hydrolysis of 4-nitrophenyl acetate by phenyl acetate. Eur J Clin Chem Clin Biochem 1992; 30(7): 391-6.

19. Kruger NJ. The Bradford method for protein quantitation. Methods Mol Biol 1994; (32): 9-15.

20. Meral I, Esrefoglu M, Dar K, Ustunova S, Aydin M, Demirtas M, et al. Effects of Nigella sativa on apoptosis and GABAA receptor density in cerebral cortical and hippocampal neurons in pentylenetetrazol induced kindling in rats. Biotech Histochem 2016; 91(8): 493-500.

21. Pearson-Smith J, Patel M. Metabolic dysfunction and oxidative stress in epilepsy. Int J Mol Sci 2017; 18(11): 2365.

22. Shi Y, Miao W, Teng J, Zhang L. Ginsenoside Rb1 protects the brain from damage induced by epileptic seizure via Nrf2/ARE signaling. Cell Physiol Biochem 2018; 45(1): 212-25.

23. Zhu X, Dong J, Han B, Huang R, Zhang A, Xia Z, et al. Neuronal nitric oxide synthase contributes to PTZ kindling epilepsy-induced hippocampal endoplasmic reticulum stress and oxidative damage. Front Cell Neurosci 2017; 11: 377.

24. Calabrese EJ. Overcompensation stimulation: a mechanism for hormetic effects. Crit Rev Toxicol 2001; 31(4-5): 425-70.

25. Ungvari Z, Parrado-Fernandez C, Csiszar A, de Cabo R. Mechanisms underlying caloric restriction and lifespan regulation: implications for vascular aging. Circ Res 2008; 102(5): 519-28.

26. Calabrese EJ, Mattson MP. How does hormesis impact biology, toxicology, and medicine? NPJ Aging Mech Dis 2017; 3(1): 13.

27. Calabrese EJ, Baldwin LA. Hormesis: a generalizable and unifying hypothesis. Crit Rev Toxicol 2001; 31(4-5): 353-424.

28. Magesh S, Chen Y, Hu L. Small Molecule Modulators of K eap1-N rf2-ARE Pathway as Potential Preventive and Therapeutic Agents. Med Res Rev 2012; 32(4): 687-726.

29. Ungvari Z, Bagi Z, Feher A, Recchia FA, Sonntag WE, Pearson $K$, et al. Resveratrol confers endothelial protection via activation of the antioxidant transcription factor Nrf2. Am J Physiol Heart Circ Physiol 2010; 299(1): H18-H24.

30. Giudice A, Arra C, Turco MC. Review of molecular mechanisms involved in the activation of the Nrf2-ARE signaling pathway by chemopreventive agents. In: Transcription factors. Methods Mol Biol 2010; (634): 37-74.

31. Kamble SM, Goyal SN, Patil CR. Multifunctional pentacyclic triterpenoids as adjuvants in cancer chemotherapy: a review. RSC Advances 2014; 4(63): 33370-82.

32. Hamdan AM, Al-Gayyar MM, Shams ME, Alshaman US, Prabahar K, Bagalagel A, et al. Thymoquinone therapy remediates elevated brain tissue inflammatory mediators induced by chronic administration of food preservatives. Sci Rep 2019; 9(1): 7026.

33. Murphy MP. Antioxidants as therapies: can we improve on nature? Free Radic Biol Med 2014; 66: 20-3.

34. Gaetani GF, Galiano S, Canepa L, Ferraris AM, Kirkman HN. Catalase and glutathione peroxidase are equally active in detoxification of hydrogen peroxide in human erythrocytes. Blood 1989; 73(1): 334-9.

35. Alam MF, Khan G, Safhi MM, Alshahrani S, Siddiqui R, Sivagurunathan Moni $S$, et al. Thymoquinone ameliorates doxorubicin-induced cardiotoxicity in swiss albino mice by modulating oxidative damage and cellular inflammation. Cardiol Res Pract 2018; 1483041 (6 pages).

36. Asgharzadeh F, Bargi R, Beheshti F, Hosseini M, Farzadnia M, Khazaei M. Thymoquinone Prevents Myocardial and Perivascular Fibrosis Induced by Chronic Lipopolysaccharide Exposure in Male Rats:-Thymoquinone and Cardiac Fibrosis. J Pharmacopuncture 2018; 21(4): 284. 
37. Horch HW, Katz LC. BDNF release from single cells elicits local dendritic growth in nearby neurons. Nat Neurosci 2002; 5(11): 1177.

38. Bathina S, Das UN. Brain-derived neurotrophic factor and its clinical implications. Arch Med Sci 2015; 11(6): 1164.

39. Altar CA, Whitehead RE, Chen R, Wörtwein G, Madsen TM. Effects of electroconvulsive seizures and antidepressant drugs on brain-derived neurotrophic factor protein in rat brain. Biol Psychiatry 2003; 54(7): 703-9.

40. Falcicchia C, Paolone G, Emerich DF, Lovisari F, Bell WJ, Fradet T, et al. Seizure-suppressant and neuroprotective effects of encapsulated BDNF-producing cells in a rat model of temporal lobe epilepsy. Mol Ther Methods Clin Dev 2018; 9: 211-24.

41. Porcher C, Medina I, Gaiarsa J-L. Mechanism of BDNF modulation in GABAergic synaptic transmission in healthy and disease brains. Front Cell Neurosci 2018; 12: 273.

42. Unsain N, Nunez N, Anastasía A, Mascó D. Status epilepticus induces a TrkB to p75 neurotrophin receptor switch and increases brain-derived neurotrophic factor interaction with p75 neurotrophin receptor: an initial event in neuronal injury induction. Neuroscience 2008; 154(3): 978-93.

43. Volosin M, Trotter C, Cragnolini A, Kenchappa RS, Light M, Hempstead BL, et al. Induction of proneurotrophins and activation of p75NTR-mediated apoptosis via neurotrophin receptor-interacting factor in hippocampal neurons after seizures. J neurosci 2008; 28(39): 9870-9.
44. Hong Z, Li W, Qu B, Zou X, Chen J, Sander J, et al. Serum brainderived neurotrophic factor levels in epilepsy. Eur J Neurol 2014; 21(1): 57-64.

45. Chen S-F, Jou S-B, Chen NC, Chuang H-Y, Huang C-R, Tsai M-H, et al. Serum Levels of Brain-Derived Neurotrophic Factor and Insulin-Like Growth Factor 1 Are Associated With Autonomic Dysfunction and Impaired Cerebral Autoregulation in Patients With Epilepsy. Front Neurol 2018; 9: 969.

46. Cosulich SC, Savory PJ, Clarke PR. BCl-2 regulates amplification of caspase activation by cytochrome c. Curr Biol 1999; 9(3): 147-50.

47. O'Brien MA, Kirby R. Apoptosis: A review of pro-apoptotic and antiapoptotic pathways and dysregulation in disease. J Vet Emerg Crit Car 2008; 18(6): 572-85.

48. Hosseini SM, Taghiabadi E, Abnous K, Hariri AT, Pourbakhsh H, Hosseinzadeh $\mathrm{H}$. Protective effect of thymoquinone, the active constituent of Nigella sativa fixed oil, against ethanol toxicity in rats. Iran J Basic Med Sci 2017; 20(8): 927.

49. Ullah I, Badshah H, Naseer MI, Lee HY, Kim MO. Thymoquinone and vitamin $C$ attenuates pentylenetetrazole-induced seizures via activation of GABA B1 receptor in adult rats cortex and hippocampus. Neuromolecular Med 2015; 17(1): 35-46.

50. Pavlova T, Stepanichev M, Gulyaeva N. Pentylenetetrazole kindling induces neuronal cyclin B1 expression in rat hippocampus. Neurosci Lett 2006; 392(1-2): 154-8. 